and both $a d w$ and $a d r$ are prevalent in the Far East, Thailand, Malaysia, Indonesia and New Guinea. The $\mathbf{R}$ zone, predominantly $a d r$, seems to be located in South East Asia and the Far East. The fourth region is the Pleotypic zone, centred in Oceania and particularly around Bougainville, as reported on page 38 of this issue of Nature by Mazzur and her colleagues. In this region practically all possible mixtures of the recognised phenotypes of hepatitis B antigen have been found.

Incursions of different subtypes into these zones and the establishment of mixed zones inevitably result from population movements. But since the subtypes are determined by the virus and not by the host, an epidemic of one strain of hepatitis B virus might result in a spate of that particular uniform strain at one time, and a later epidemic of a different subtype might leave carriers of another subtype even in the same area. This has been recorded in Stockholm, for example. Magnius and his associates (Scand. J. infect. Dis., 5, 81; 1973) examined stored sera from seventy-two patients with viral hepatitis admitted to a hospital in Stockholm in 1953-54 and sera from 202 patients with hepatitis admitted in 1970 . Hepatitis $B$ antigen was detected in $43 \%$ and $49 \%$ of the patients respectively. The subtypes from these two groups and from ninety-five antigen-positive drug addicts were determined. Twenty-two $(71 \%)$ of thirty-one patients from the 1953-54 group were of ad subtype whereas only two were ay (seven samples could not be typed). In 1970, however, seventy-five $(76 \%)$ of ninety-nine patients were ay whereas only twelve were ad (the remaining twelve sera could not be subtyped). Ninety $(95 \%)$ of the antigen-positive drug addicts were ay and five were ad. A shift has thus occurred in Stockholm since 1953-54 from predominantly ad to the ay subtype. It is speculated that the tourist influx from Northern Europe to the Southern Mediterranean countries, the Middle East and North Africa, an ay zone, as well as the attraction of these countries to drug addicts may have been factors in the shift of hepatitis B antigen subtypes noted in Stockholm.

The latest report by Mazzur and her associates in the current issue of Nature also confirms the varied geographical distribution of antigen subdeterminants $d, y$ and $w$, and, although the antigen mapping of the world is fragmentary and far from complete, information is rapidly accumulating. Hepatitis $B$ antigen has previously been shown to be a useful epidemiological marker; it may well prove to be a fascinating ecological marker of both ancient and more recent population migration.

From our Medical Virology Correspondent

\section{Plant diversity in grassland}

from our Plant Ecology Correspondent The publication by the Kent Trust for Nature Conservation of papers given at a conference on chalk grassland management held a year ago at the King's College Field Centre, Rogate, Hampshire, will reopen the question of what determines plant species diversity in this habitat (see Nature, 240, 443; 1972). It may also raise new questions regarding the objectives of management practices and priorities in conservation.

The diversity issue has been clarified by Grime (Nature, 242, 344; 1973), who has come to the conclusion that diversity in grassland habitats is clearly related to environmental stresses. If stress is low, then grasslands become dominated by species of high physical competitive ability, that is species which grow rapidly, are robust and produce dense litter. Stress reduces the performance of these species, allowing other species to enter the community as a result of the lowering of competition. Diversity thus increases with stress until the stress becomes so severe that only species which are specifically adapted to that particular adverse factor are capable of survival. Then diversity falls. Some authors emphasise the role of predation in the maintenance of vegetation diversity (for example, Harper, Brookhaven Symp. Biol., 22, 48; 1969) whereas others feel that nutrient availability may be the critical factor (for example, Green, Biol. Conserv., 4, 378; 1972).

In the recently published report (Chalk Grassland, ed. by Jermy and Stott, Kent Trust for Nature Conservation; 1973), both sides of the argument are represented. Wells considers that the composition of a grassland community can best be understood by reference to the respective life forms and growth periods of its constituent species. In other words, if mowing or grazing stresses are applied to a grassland then the species which lose the smallest proportion of their above ground biomass will be at a selective advantage. Tall, robust species are eliminated to the advantage of short-growing species, thus allowing the development of a diverse assemblage of species with an average $67 \%$ of low-growing hemicrytophytes.

Harding defends the point of view that nutrient availability limits the growth of aggressive species. Using Shannon's diversity index, he demonstrates a negative correlation between angiosperm diversity in the grass sward and total nitrogen in the soil. $\mathrm{He}$ follows Green in the belief that a buildup of nitrogen in the soil permits invasion and attainment of dominance by robust species, thus reducing overall diversity. It is always dangerous, how- ever, to infer causation from correlation. One could equally well argue that the presence of robust, dominant species causes a build-up of nitrogen in the soil by rapid litter production and hence the encouragement of soil microbiol activity, including nitrogen fixation. Suspension of robust species by applying a grazing stress could keep soil nitrogen levels low. The nutrient argument still lacks direct experimental support.

The quest of conservationists for biotic diversity becomes even more complex when one considers the zoological aspect of the problem. For invertebrates in particular, microclimatic diversity (which is a function of vegetation structural complexity) is an overriding influence upon the potential faunal diversity. It is not surprising, therefore, that Morris demonstrates a positive correlation between the number of species of Auchenorhyncha and the vegetation height at the sampling sites. It does, however, pose a management problem, since in selecting a management regime which determines vegetation height, one must choose between floral and faunal diversity.

\section{Proteins and odour perception in insects}

\section{from our Insect Physiology Correspondent}

THERE have been several suggestions that enzymatic processes are involved in olfactory perception in insects. Schneider observed that the antennae of the male silkworm rapidly degrade the sex pheromone bombycol following stimulation; and Kasan and Kaissling confirmed that bombycol, (E)-10-(Z)-12-hexadecadien1 -ol, was progressively degraded into acidic and ester fractions by enzymes in the antennae and other parts of the body. In this way the receptors quickly become competent once more to receive the olfactory stimulus. Norris, Ferkovich and others have shown that naphthoquinones, which function as feeding inhibitors in the cockroach Periplaneta, exert their activity by binding to sulphydryl groups of specific antennal proteins.

Ferkovich, Mayer and Rutter ( $J$. Insect Physiol., 19, 2231; 1973) have now studied the sex attractant of the cabbage looper, Trichoplusia ni, along the same lines. This pheromone is $(\mathrm{Z})$ 7-dodecen-1-ol acetate. When exposed to a soluble protein present in the male antennae it suffers enzymatic conversion to the alcohol (Z)-7-dodecen-1-ol-which not only lacks sex attractancy but is a potent inhibitor of behavioural responses to the normal pheromone. The protein responsible for enzymatic hydrolysis of the pheromone exists in smaller amounts elsewhere in the body, but it is most active in the antennae. The inhibitory 